\title{
Derivation and transcriptional profiling analysis of pluripotent stem cell lines from rat blastocysts
}

\author{
Chunliang $\mathrm{Li}^{1,4,{ }^{*}}$, Ying Yang ${ }^{1,4,}$, Junjie $\mathrm{Gu}^{1,2,3}, \mathrm{Yu} \mathrm{Ma}^{1,2,3}$, Ying Jin ${ }^{1,2,3}$ \\ ${ }^{1}$ Key Laboratory of Stem Cell Biology, Institute of Health Sciences, Shanghai Institutes for Biological Sciences, Chinese Academy \\ of Sciences/Shanghai Jiao Tong University School of Medicine; 225 South Chongqing Road, Shanghai 200025, China; ${ }^{2}$ Shanghai \\ Stem Cell Institute, Shanghai Jiao Tong University School of Medicine, Shanghai 200025, China; ${ }^{3}$ Key Laboratory of Cell Differ- \\ entiation and Apoptosis of Chinese Ministry of Education, Shanghai Jiao Tong University School of Medicine, Shanghai 200025, \\ China; ${ }^{4}$ Graduate School of Chinese Academy of Sciences, Beijing 100049, China
}

Embryonic stem (ES) cells are derived from blastocyst-stage embryos. Their unique properties of self-renewal and pluripotency make them an attractive tool for basic research and a potential cell resource for therapy. ES cells of mouse and human have been successfully generated and applied in a wide range of research. However, no genuine ES cell lines have been obtained from rat to date. In this study, we identified pluripotent cells in early rat embryos using specific antibodies against markers of pluripotent stem cells. Subsequently, by modifying the culture medium for rat blastocysts, we derived pluripotent rat ES-like cell lines, which expressed pluripotency markers and formed embryoid bodies (EBs) in vitro. Importantly, these rat ES-like cells were able to produce teratomas. Both EBs and teratomas contained tissues from all three embryonic germ layers. In addition, from the rat ES-like cells, we derived a rat primitive endoderm (PrE) cell line. Furthermore, we conducted transcriptional profiling of the rat ES-like cells and identified the unique molecular signature of the rat pluripotent stem cells. Our analysis demonstrates that multiple signaling pathways, including the BMP, Activin and mTOR pathways, may be involved in keeping the rat ES-like cells in an undifferentiated state. The cell lines and information obtained in this study will accelerate our understanding of the molecular regulation underlying pluripotency and guide us in the appropriate manipulation of ES cells from a particular species.

Keywords: embryonic stem cells, blastocysts, primitive endoderm, teratomas

Cell Research (2009) 19:173-186. doi: 10.1038/cr.2008.301; published online 4 November 2008

\section{Introduction}

Pluripotent mouse embryonic stem (ES) cells are derived from the inner cell mass (ICM) of blastocyst-stage embryos [1] and they have been proven capable of indefinite self-renewal and of retention of their pluripotent potential to form all cell types in vitro [2-4]. In addition, they can be genetically engineered and can contribute to all three embryonic germ layers and to the germ line in chimeras when injected into host blastocysts, offering a

*These two authors contributed equally to this work.

Correspondence: Ying Jin

Tel/Fax: 86-21-63852591

E-mail: yjin@sibs.ac.cn

Received 10 August 2008; revised 6 October 2008; accepted 15 October 2008; published online 4 November 2008 unique opportunity to produce live mice with a desired mutation in every cell [5]. Therefore, mouse ES cells have served as a valuable model and an important tool in studying mammalian development. More recently, human ES cell lines were successfully generated, making ES cells also an attractive source for cell therapy [6].

Apart from ES cells from mouse and human, scientists have made considerable efforts to generate pluripotent ES cell lines from different vertebrate species, in particular, from rat [7-9], an important model for biomedical research and for study of human diseases. However, the attempts to establish genuine ES cell lines from rat have been unsuccessful to date. Several reports describe "rat ES-like cells", which grew like ES cells in culture [812]. However, the developmental capacity of these cells was not well characterized in vivo. It is worth noting that in the published reports, rat ES-like cells were derived 
under similar conditions to those used for generation of mouse ES cells. In fact, differences in intrinsic biology between mouse and rat have been observed [13]. Therefore, understanding the molecular mechanisms of pluripotency in rat embryonic pluripotent cells is an essential step towards successful derivation and application of rat ES cells. Nevertheless, there has been a lack of transcriptional profiling of rat pluripotent cells and we have accumulated little information about optimal conditions for derivation and maintenance of rat ES cells in culture.

In this study, we have successfully derived and characterized rat ES-like cell lines, which not only express pluripotency markers and form well-organized embryoid bodies (EBs), but also produce various cell types of the three embryonic germ layers in EBs and teratomas. In addition, we generated a rat primitive endoderm (PrE) cell line from the rat ES-like cells. To identify the molecules uniquely expressed in the undifferentiated rat ESlike cells, we performed transcriptional profiling of the rat ES-like cells and PrE cells. The molecular signature of the undifferentiated rat ES-like cells has been carefully analyzed. The cell lines and information obtained in this study will accelerate our understanding of molecular mechanisms of pluripotency and guide us in the proper manipulation of ES cells from a particular species.

\section{Results}

\section{Localization of pluripotent cells in rat early embryos}

Mouse ES cells have been successfully derived from the ICM of blastocysts expressing Oct4, Nanog and Sox2. However, the localization of pluripotent cells expressing these marker genes in rat early embryos is poorly characterized. To isolate rat ES cells, we began by examining the localization of Oct4-expressing cells in rat embryos at the blastocyst stage (4.5 day post coitus, dpc). Immunofluorescent imaging revealed that Oct4-staining was restricted in the ICM, but not in the
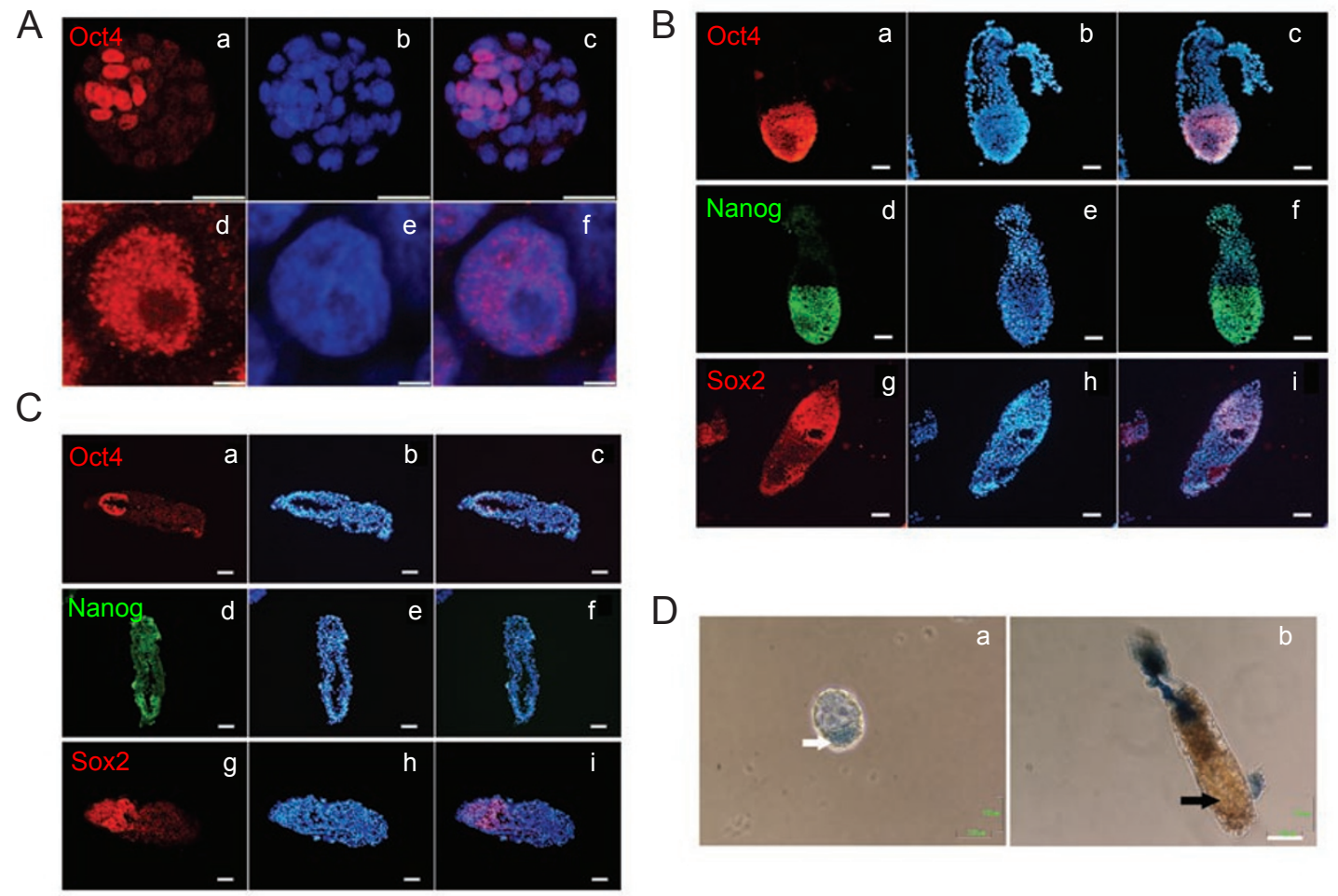

Figure 1 Localization of pluripotent cells in early rat embryos. (A) The rat Oct4 protein (red) is detected in the ICM of $4.5 \mathrm{dpc}$ rat blastocyst (a). Punctate staining of Oct4 is observed in all nuclei (absent in nucleoli) (d). DAPI staining (blue) highlighting the nuclei is shown in panels $b$ and e. The composite images are shown in panels $c$ and $f$. The scale bars in a-c are $25 \mu \mathrm{m}$ and in d-f are $2.5 \mu \mathrm{m}$. (B) Expression of pluripotency markers, Oct4 (red, a), Nanog (green, d) and Sox2 (red, g), is detected in the epiblast of rat $7.5 \mathrm{dpc}$ embryo at egg cylinder stage. Corresponding DAPI staining is shown in panels $b$, e and $h$. The composite images are shown in panels c, $\mathrm{f}$ and $\mathrm{i}$. The scale bars are $50 \mu \mathrm{m}$. (C) Expression of Oct4 (red, a), Nanog (green, d) and Sox2 (red, g) is examined in the mouse $6.0 \mathrm{dpc}$ embryo at egg cylinder stage. Corresponding DAPI staining is shown in panels $b$, e and $h$. The composite images are shown in panels $c, f$ and $i$. The scale bars are $50 \mu m$. (D) AKP staining is positive in the ICM of the rat $4.5 \mathrm{dpc}$ embryo at the blastocyst stage (a, white arrow). No signal is observed in the epiblast of the rat $7.5 \mathrm{dpc}$ embryo at the egg cylinder stage (b, black arrow). The scale bars are $100 \mu \mathrm{m}$. 
A

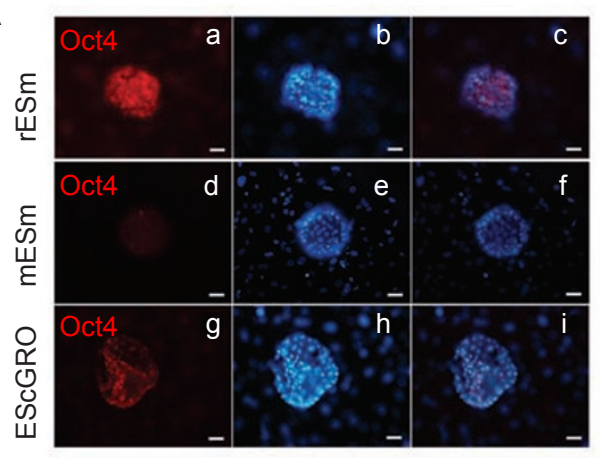

B

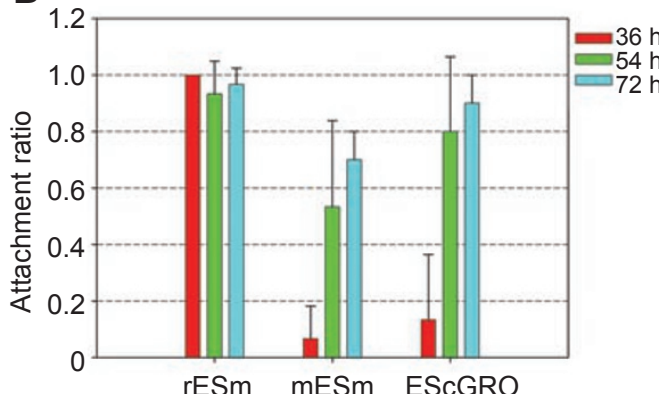

C
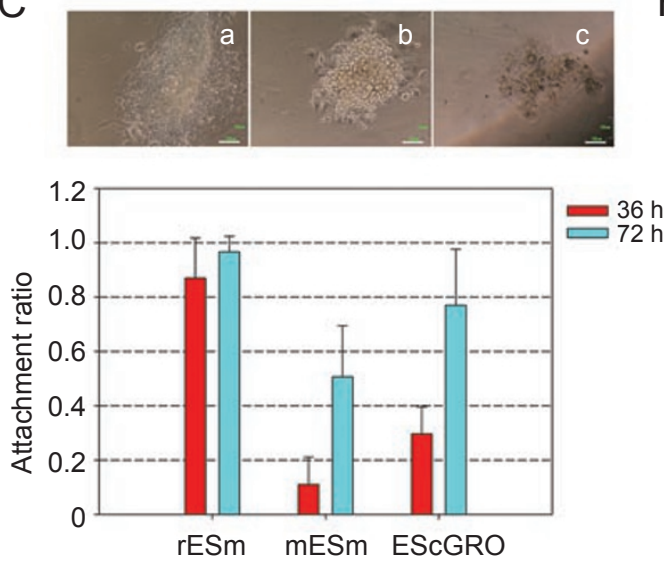

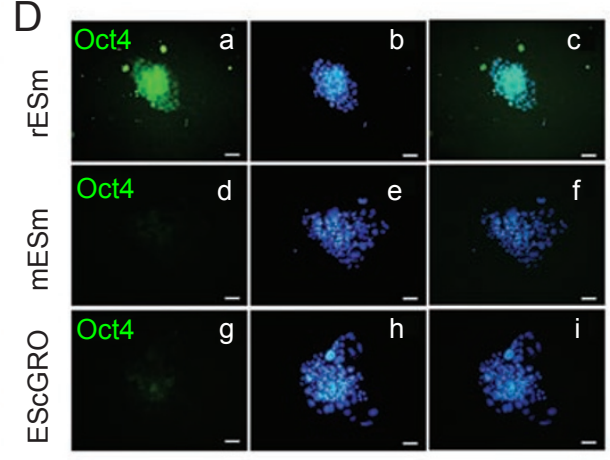

E

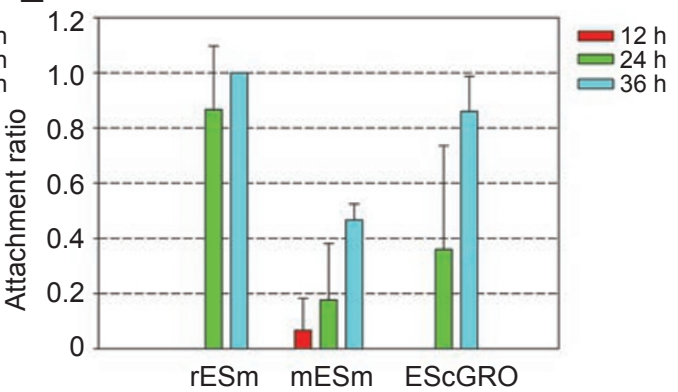

F

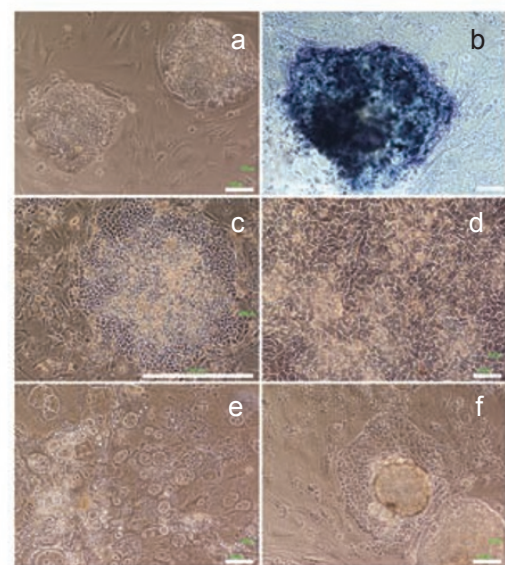

Figure 2 Identification of the optimal medium for derivation of the rat ES-like cells. (A) Whole rat blastocysts were cultured in rat ES cell medium (a-c), mouse ES cell medium (d-f) and EScGRO ( $g-i)$. Oct4 (red) expression was detected by immunostaining after $72 \mathrm{~h}$ in primary culture. Corresponding DAPI staining is shown in panels $\mathrm{b}$, e and h. The composite images are shown in panels c, f and i. The scale bars are $50 \mu \mathrm{m}$. (B) While rat blastocysts were cultured under feeder-dependent conditions, their attachment ratio in different culture media was quantified. The columns display the attachment ratio. The error bar displays standard deviation from three independent experiments. (C) When rat blastocysts were cultured under feederfree conditions, images of their morphology were captured in the rat ES cell medium (a), the mouse ES cell medium (b) and EScGRO (c). The attachment ratio of rat blastocysts in the different culture media was quantified (lower panel). The columns display the attachment ratio. The error bar displays standard deviation from three independent experiments. The scale bars are $100 \mu \mathrm{m}$. (D) Zona pellucida free rat blastocysts were cultured in the rat ES cell medium (a-c), mouse ES cell medium (df) and EScGRO (g-i). Oct4 (green) expression was detected by immunostaining after $12 \mathrm{~h}$ in primary culture. Corresponding DAPI staining is shown in panels $b$, e and $h$. The composite images are shown in panels $c$, $f$ and $i$. The scale bars are $50 \mu \mathrm{m}$. (E) When zona pellucida free rat blastocysts were cultured under feeder-dependent conditions, their attachment ratio in the different culture media was quantified. The columns display the attachment ratio. The error bar displays standard deviation from three independent experiments. (F) The mechanically passaged rat ES-like cells maintain typical morphology of an ES cell colony (a) and are positive for AKP staining (b). The enzymatically passaged rat ES-like cells maintain a similar morphology (c-d). The differentiated trophoblast giant cells (e) and endoderm-like cells ( $f$ ) are observed in the periphery of the partially differentiated rat ES-like cell colonies. The scale bar in c is $1000 \mu \mathrm{m}$ and the rest are $100 \mu \mathrm{m}$. 
peripheral trophectodermal cells (Figure 1A). The staining of Oct4 in the nuclei of the ICM cells was punctate, being absent from the nucleoli. Furthermore, we checked the localization of pluripotent cells in rat embryos at 7.5 dpc. Mouse embryos at $6.0 \mathrm{dpc}$ were also examined as a control. As shown in Figure 1B, the positive signals for Oct4, Nanog and Sox 2 were all localized in the epiblast of rat embryos. In contrast, extra-embryonic tissues were negative for all three antibodies. Moreover, staining for endoderm markers, Gata4 and $\mathrm{Hnf} 4 \alpha$, was positive in rat extra-embryonic endoderm cells, but not in the epiblast cells (Supplemental imformation Figure S1). Similar to rat embryos, Oct4-, Nanog- and Sox2-positive cells were detected in the epiblast of mouse embryos (Figure $1 C)$, consistent with previous reports $[14,15]$. These observations indicate that embryonic pluripotent cells are localized in the ICM and in the epiblast of early rat embryos. To further confirm the localization of the pluripotent cells, expression of another pluripotency marker, alkaline phosphatase (AKP), was examined in the ICM of blastocyst and the epiblast of the egg cylinder of rat embryos (Figure 1D). Our results show that AKP staining was positive in the ICM cells, but became negative in the epiblast of the egg cylinder, consistent with the AKP staining pattern seen in mouse embryos $[16,17]$.

\section{Optimization of culture medium for derivation of rat ES-} like cells

Knowing that the expression pattern of pluripotency markers in early mouse and rat embryos is similar (Figure 1), we tried to derive rat ES cell lines utilizing a medium similar to that used for mouse ES cell derivation. However, after rat blastocysts were plated onto mouse embryonic fibroblast (MEF) cells, the ICM cells did not grow well in either mouse ES cell medium or in the component defined medium (EScGRO, Millipore). Further study showed that rat blastocysts cultured in the mouse ES cell medium readily differentiated into trophoblast giant cells, while embryos cultured in the EScGRO markedly differentiated into endoderm-like cells (data not shown). The phenomenon suggested to us that the mouse ES cell medium might contain factors inhibitory to primitive endoderm commitment and that the EScGRO might contain components inhibitory to trophoblast giant cell differentiation. We thus hypothesized that the combination of the two media might promote ICM cell proliferation in an undifferentiated state. To test the hypothesis, we mixed the mouse ES cell medium with EScGRO at a 1:1 ratio and included in the mixture $2 \mu \mathrm{M}$ GSK-3 selective inhibitor (BIO), which had been demonstrated to help maintain both mouse and human ES cells in an undifferentiated state in culture [18, 19]. We named the mixture "rat ES cell medium". When rat embryos cultured for 5 days were stained with antibody against Oct4, we observed significantly more Oct4-positive cells using the rat ES cell medium as compared to cultures using either the mouse ES cell medium or EScGRO (Figure 2A). To determine why more Oct4-postive cells were obtained with the rat ES cell medium, we compared the attachment ratio of rat blastocysts in the three culture media. After whole blastocysts were plated in the rat ES cell medium, almost $100 \%$ of blastocysts attached to MEF cells within $36 \mathrm{~h}$. However, less than $20 \%$ were found to attach either in the mouse ES cell medium or EScGRO. Even after $72 \mathrm{~h}$, only approximately $70 \%$ and $90 \%$ of blastocysts attached in the mouse ES cell medium and EScGRO, respectively (Figure 2B). When the same experiments were repeated in matrigel-coated culture plates, obvious improvement of the embryo attachment ratio was also obtained with the rat ES cell medium, particularly within $36 \mathrm{~h}$ (Figure 2C). We believe that acceleration of attachment of blastocysts is the key factor contributing to the greater number of Oct4-positive cells observed in the rat ES cell medium. To further confirm this assumption and exclude the influence of zona pellucida on hatching, we treated the whole rat blastocysts with Tyrode's solution to destroy the zona pellucida and repeated the embryo attachment experiment. Again, more Oct4-positive cells

Table 1 Derivation Efficiency of rat ES-like cell lines

\begin{tabular}{|c|c|c|c|c|c|}
\hline Experiment & No. of blastocysts & Cell line & Name & Teratoma (three germ layer) & Efficiency \\
\hline I & 11 & 1 & rES1222 & Yes/intramuscular injection & $9 \%$ \\
\hline II & 8 & 1 & rES0107 & Yes/subretinal injection & $12.5 \%$ \\
\hline \multirow[t]{2}{*}{ III } & 8 & 3 & rES1122 & N.D. & $37.5 \%$ \\
\hline & & & rES0113 & Yes/subretinal injection & \\
\hline Total & 31 & 6 & & & $19 \%$ \\
\hline
\end{tabular}


A

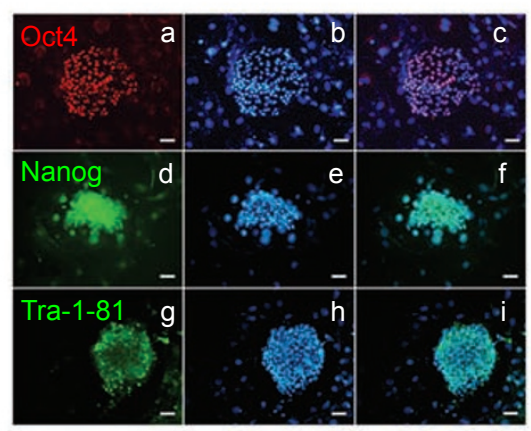

B

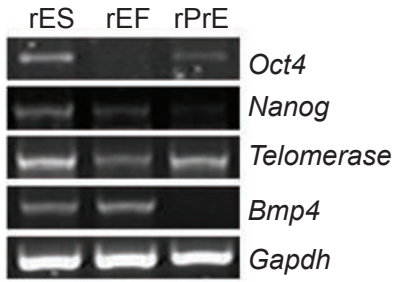

$\frac{\infty}{0}$

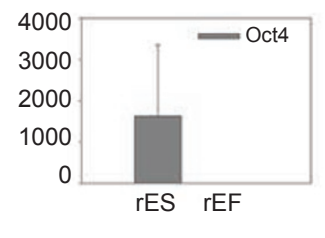

C
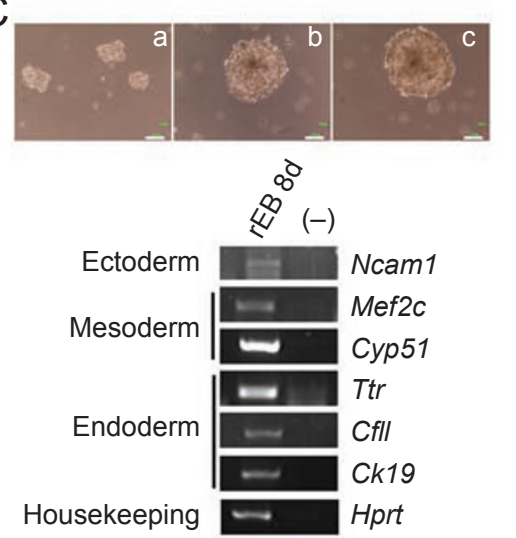

$E$

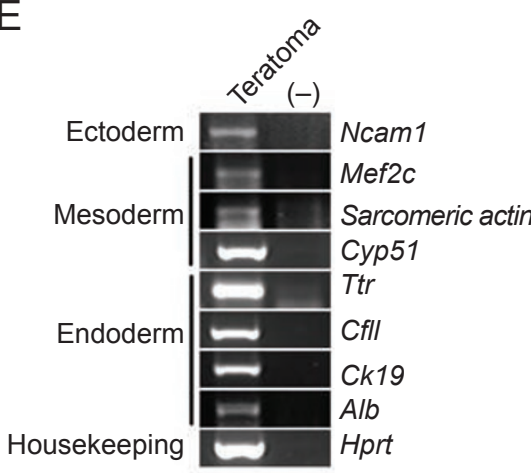

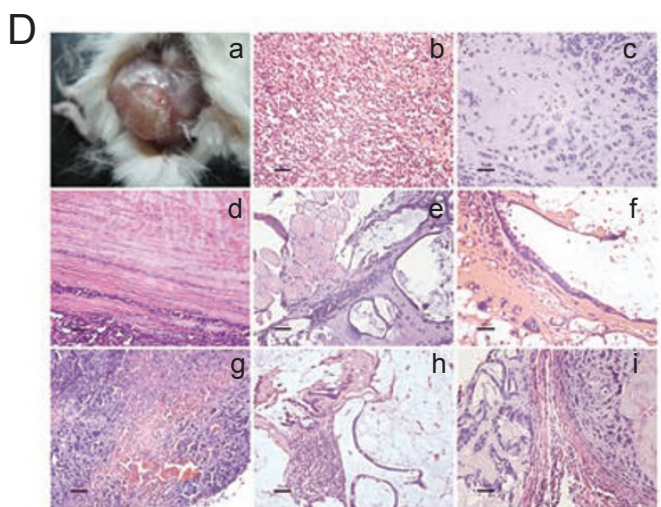

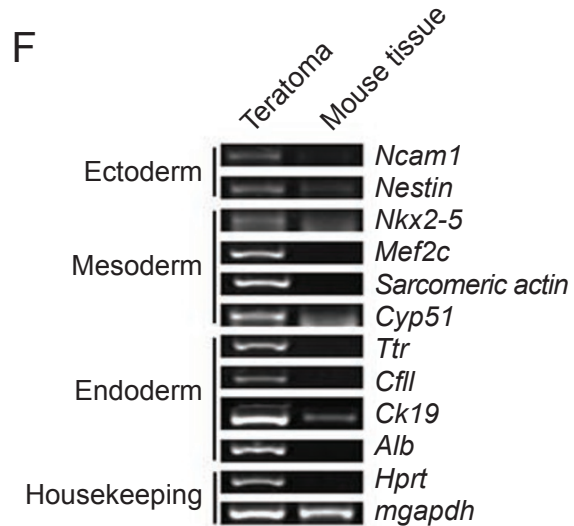

Figure 3 Characterization of rat ES-like cells in vitro and in vivo. (A) The cultured rat ES-like cells at passage 7 are Oct4- (red, a), Nanog- (green, d) and Tra-1-81- (green, g) positive. Corresponding DAPI staining is shown in panels b, e and h. The composite images are shown in panels $\mathrm{C}, \mathrm{f}$ and $\mathrm{i}$. The scale bars are $50 \mu \mathrm{m}$. (B) Expression of pluripotency marker genes in rat ES-like cells was examined by the RT-PCR analysis at passage 12 and compared with expression levels in rEF cells and rat PrE cells. Quantitative real time PCR analysis for expression of the rat Oct4 level was done in different passages of rat ESlike cell lines 3151 and 1222. The error bar displays standard deviation from three independent experiments. (C) The rat ESlike cells form cystic EBs in serum containing medium (a). The PrE cells appear in the periphery of EBs from day 7 (b) and EBs grow significantly at day 9 (c). The scale bars are $100 \mu \mathrm{m}$. The RT-PCR analysis of the three germ layer markers confirms the existence of various tissues in the EBs formed from rat ES-like cells. (D) Teratomas were harvested 4 months after intramuscular injection of the rat ES-like cells into SCID mice (a). Various tissues such as myelinated nerve fiber (b); cartilage (c); smooth muscle (d); fat (e); columnar epithelium of lung (f); blood vessel (g); goblet cells (h) and skeletal muscle (i) were detected by histochemical staining. The scale bars are $50 \mu \mathrm{m}$. (E) RT-PCR analysis of the three germ layer markers confirms the existence of various tissues in the teratomas derived from the rat ES-like cells. (F) RT-PCR analysis of the three germ layer markers confirms the specificity of the primers used for the analysis of rat genes in teratomas. 
and a significant improvement of the embryo attachment ratio were obtained with the rat ES cell medium, although embryos attached to the matrigel much sooner in the absence of the zona pellucida than in its presence for all three media (Figure 2D-2E). These observations favor our conclusion that the rat ES cell medium has an ability to enhance embryo attachment during rat ES cell derivation. With the enhanced blastocyst attachment in the rat ES cell medium, we were able to mechanically passage the rat ES-like cells in the early five passages. Undifferentiated rat ES-like cells with a high nucleus to cytoplasm ratio formed compacted colonies. The periphery of the rat ES-like colony was distinct from MEF cells and the rat ES-like cells were AKP-positive (Figure 2F, a, b). When passaged with trypsin, approximately $50-70 \%$ of cells displayed undifferentiated cell morphology, which appeared homogenous with a high nucleus to cytoplasm ratio (Figure $2 \mathrm{~F}, \mathrm{c}, \mathrm{d}$ ). The rest of the cells, especially those in the periphery of the colonies, exhibited differentiated morphology. Two types of differentiated morphology were found, one resembling trophoblast giant cells (Figure 2F, e), and the other resembling endoderm cells (Figure 2F, f). The trophoblast giant cells gradually vanished during continuous culture, but the endoderm-like cells remained in the partially differentiated colonies. At present, six rat ES-like cell lines from thirty-one rat blastocysts have been generated (Table 1) and one of them (line 3151) has been continuously passaged for four months (more than twenty passages). Moreover, the cells can be frozen and thawed successfully.

\section{Characterization of rat ES-like cell lines}

One of the criteria for pluripotent stem cells is their expression of a group of pluripotency marker genes. As shown in Figure 3A, the nuclear localization of Oct4, Nanog and membrane localization of Tra-1-81 were observed in the undifferentiated rat ES-like cells (two independently derived rat ES-like cell lines, line 1222 and line 3151). In contrast, MEF cells were negative for these antibodies. Due to the limited availability of antibodies reactive to rat antigens, we also collected RNA samples from the rat ES-like cells, rat embryonic fibroblast (REF) cells and rat primitive endoderm (PrE, see following section) cells to determine expression levels of several marker genes (Figure 3B). RT-PCR analysis showed that Oct4 and Nanog were highly expressed in the undifferentiated rat ES-like cells, whereas their expression in the rat PrE and REF cells was weak. Quantitative real time PCR (qPCR) analysis further confirmed distinctly higher expression of Oct4 in rat ES-like cells as compared to REF cells (Figure 3B, bottom panel). Furthermore, the expression level of the rat Tert gene was highest in the rat
ES-like cells. Modest and weak expression of the rat Tert gene was detected in the rat PrE and REF cells, respectively. Bmp4 was reported as an important cytokine mediating the pluripotent state and mesoderm differentiation in mouse ES cells $[20,21]$. We found high expression of Bmp4 in the rat ES-like cells as well as in the REF cells.

The second hallmark of ES cells is their potential to form all cell types of the three embryonic germ layers both in vitro and in vivo. The in vitro differentiation potential of mouse and human ES cells has often been tested using the EB formation assay. Our rat ES-like cells formed EBs as mouse and human ES cells usually do. After culture for 6-8 days, two distinct layers were observed in the EBs, the interior layer in the center and the outer layer on the periphery of the EBs (Figure 3C). A similar phenomenon was always observed in mouse EBs, in which the outer layer primarily contains PrE cells [22]. RT-PCR analysis confirmed the presence of markers for the three germ layers in our EBs, such as Ncam1, Mef2c, Cyp51, Ttr, CfII and Ck19 (Figure 3C, bottom panel). This observation demonstrates that our rat ES-like cells have the ability to differentiate normally in vitro. To test their differentiation ability in vivo, a teratoma formation assay was performed by intramuscular methods for the rat ES cell lines 1222 and 3151 in SCID mice. Four months after injection, teratomas were collected from recipient mice (Figure 3D, a). In the teratoma sections, we observed typical myelinated nerve fiber (ectoderm), cartilage, smooth muscle, skeletal muscle, fat (mesoderm) and columnar epithelium of lung (endoderm) (Figure $3 \mathrm{D}, \mathrm{b}-\mathrm{i})$. In addition, the expression of marker genes for all three germ layers was readily detected in the RNA samples extracted from the rat ES-like cell-derived teratomas, but not in the RNA samples which were not reversely transcribed, revealing that the RT-PCR signals originated from the RNA in our samples, but not from potentially contaminating genomic DNA (Figure 3E). To ensure the specificity of the PCR primers used for the rat samples, we assessed the gene expression levels in the rat ES cell-derived teratomas and mouse tissues, using the same primers. As shown in Figure 3F, the primers exclusively recognized the rat RNA extracted from teratomas, but did not react to the RNA extracted from mouse tissues. These data provide strong evidence that our rat ES-like cells have pluripotent differentiation potential. In addition, three other rat ES-like cell lines also generated teratomas using subretinal injection methods (data not shown).

\section{Rat PrE cell line derived from rat ES-like cells}

As mentioned above, there were PrE-like cells in the periphery of the partially differentiated colonies (Figure 
A
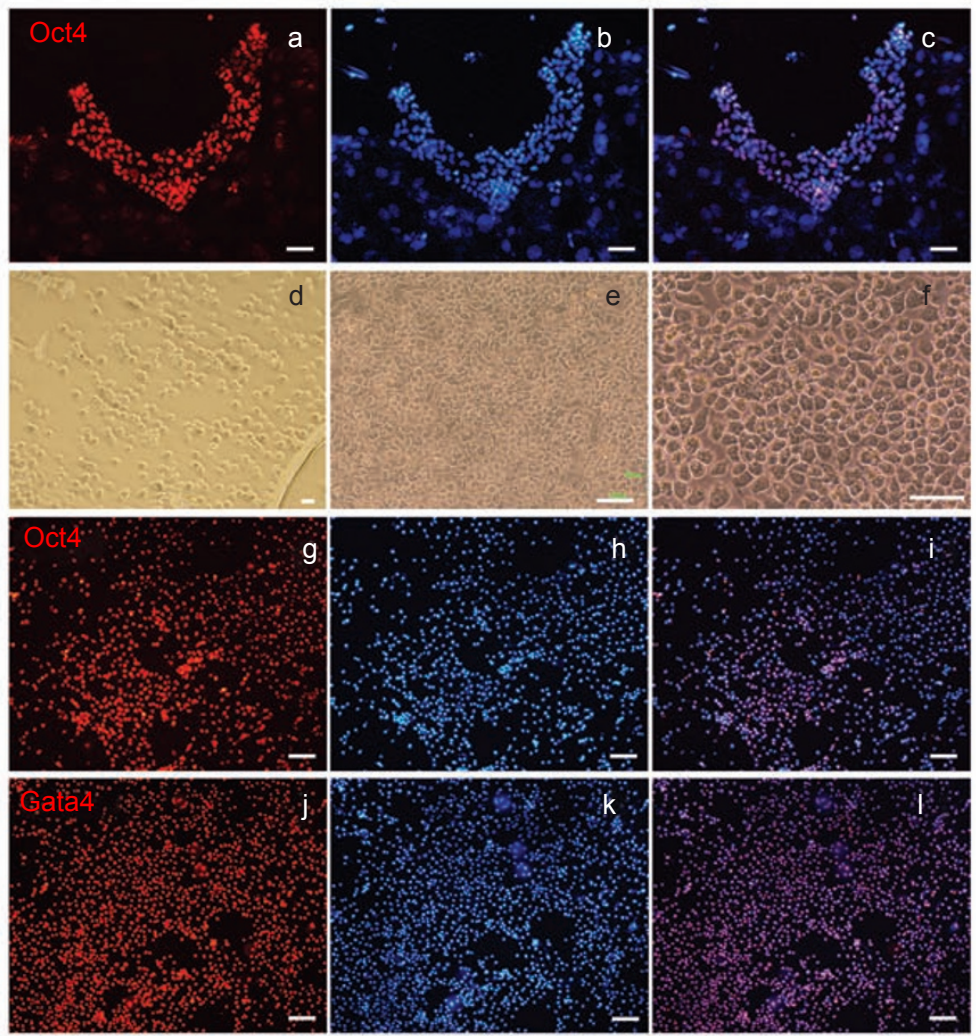

B
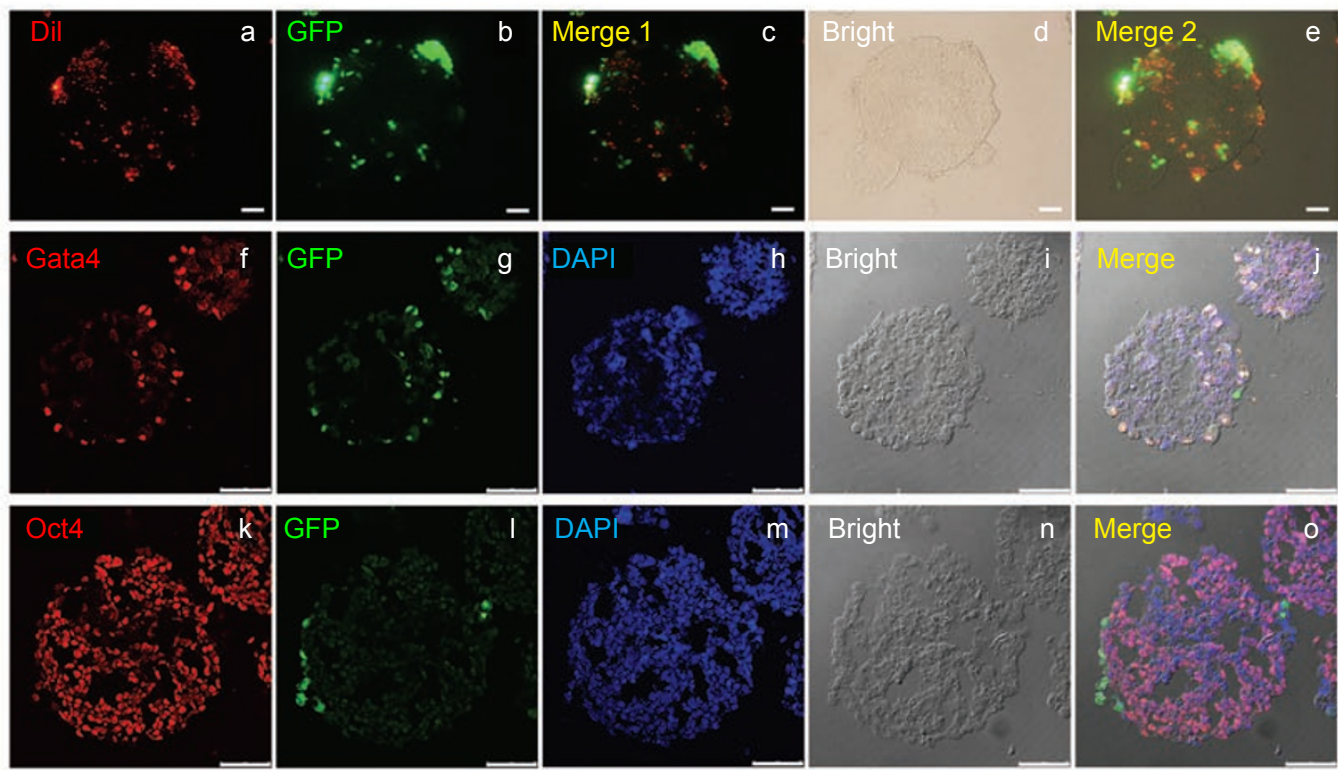

Figure 4 Characterization of rat PrE cells differentiated from rat ES-like cells. (A) The endoderm-like cells exist in the periphery of the partially differentiated rat ES-like colony. After the undifferentiated rat ES-like cells in the center of the colony were mechanically removed, the remaining endoderm-like cells located in the periphery of the colonies were stained by Oct4-antibody (red, a). When these differentiated cells were transferred to the gelatin-coated culture dish and passaged continuously (d-f), they were seen to be positive for Oct4 (red, g) and Gata4 (red, j) by immunostaining at passage 5. Corresponding DAPI staining is shown in panels $\mathrm{b}, \mathrm{h}$ and $\mathrm{k}$. The composite images are shown in panels $\mathrm{c}, \mathrm{i}$ and $\mathrm{I}$. The scale bars in a-c are $50 \mu \mathrm{m}$ and in $\mathrm{d}-\mathrm{I}$ are $100 \mu \mathrm{m}$. (B) The GFP and Dil double-labeled rat PrE cells at passage 7 are seen to migrate to the periphery of chimeric EB when mixed with mouse ES cells (a-e). These GFP labeled rat PrE cells express Gata4 (red, f) and Oct4 (red, k) as detected by immunostaining. The scale bars in a-e are $50 \mu \mathrm{m}$ and in f-o are $75 \mu \mathrm{m}$. 
2F, f). Interestingly, these cells expressed Oct4 (Figure 4A, a). To characterize the PrE-like cells in detail, we derived the rat $\mathrm{PrE}$ cell line by mechanically transferring the PrE-like cells onto gelatin-coated cell culture dishes and culturing the cells in the mouse ES cell medium in the absence of leukemia inhibitory factor (LIF), a factor routinely used in mouse ES cell culture. We were able to passage these cells in vitro for more than 10 passages using trypsin (Figure 4A, d-f). The cells stably maintained PrE cell morphology. Immunostaining also showed that Oct 4 could be detected in almost all the cells examined (Figure 4A, g), as could the endoderm marker Gata4 (Figure 4A, j). To test whether the rat PrE cells behaved like mouse PrE cells, we conducted the chimeric EB formation assay, which has been shown to be effective in determining whether the tested cells are of PrE origin. The assay is based upon the phenomenon that PrE cells exclusively migrate to the surface of EBs when mixed with undifferentiated mouse ES cells in suspension culture [23]. Thus, we mixed rat PrE cells (double labeled with GFP and DiI dye) with unlabeled mouse ES cells. As expected, almost all the GFP and DiI double labeled rat PrE cells became positioned in the periphery of the chimeric EBs (Figure 4B, a-e), demonstrating that rat PrE cells have the ability to migrate and integrate into the outer layer of EBs, as would mouse PrE cells. However, when we mixted rat ES-like cells stained by DiI with the mouse ES cells, we could see the even distribution of the rat ES-like cells throughout the chimeric EBs (data not shown). Furthermore, we examined the spatial expression pattern of Oct4 and Gata4 in the chimeric EBs. Clearly, Gata4-positive cells were co-localized with GFP-labeled PrE cells in the periphery of the EBs (Figure 4B, f-j). However, Oct4 was found in both interior cells and the outer layer of the EBs (Figure 4B, k-o), consistent with our observation that Oct4 is expressed in both the undifferentiated rat ES-like cells and the PrE cells. These data provide the functional evidence for the identity of our derived PrE cell line.

\section{Genome-wide transcriptional profiling of rat ES-like} cells

To identify specifically expressed genes in the undifferentiated rat ES-like cells, we performed transcriptional profiling of these cells using Affymetrix rat expression array 230 2.0. The Chip included 31099 probe sets, in which 11993 probe sets were related to ESTs. The rat PrE cell line was chosen as the reference, since these cells were observed in the culture of rat ES-like cells. 629 transcripts were selected as genes enriched specifically in the undifferentiated rat ES-like cells based upon up-regulation of their expression level by more than 2-fold in the rat ES-like cells as compared to the level in the PrE cells and based upon their detection at significant levels (Affymetrix "P" call). Among them, 418 transcripts had the gene symbol and the other 211 transcripts were related to ESTs (Supplementary information, Table S1). The transcripts with annotation which were upregulated by more than 10 -fold in the rat ES-like cells are shown in Figure 5A.

All the rat ES-like cell-enriched genes were classified according to the functional annotation on NetAffx analysis center (https://www.affymetrix.com/analysis/netaffx/ index.affx) (Figure 5B and Supplementary information, Table S1). Among the 629 transcripts, 303 (48\%) were related to transcripts of unknown function and ESTs. Surprisingly, for the other 326 transcripts with functional annotation, the activity in regard to maintaining ES cell pluripotency was not found for most of them in the PubMed database. Nevertheless, there were molecules which are known to be highly expressed in undifferentiated human or mouse ES cells, such as Thy1, Fbxo15, $G b \times 2, D n m t 3 a$ and Dnmt3b, which were also enriched in the rat ES-like cells. In fact, many transcription factors were enriched (9\%). Klf2, encoding a Krüppel-like factor, was highly expressed in the rat ES-like cells. Core Klf circuitry including Klf2, Klf4 and Klf5 has been reported to have a synergistic effect on self-renewal of mouse ES cells and to regulate key pluripotency genes, including Nanog [24]. The high expression level of Klf2 implies a role for the Klf molecule in rat ES-like cells. Moreover, the other two groups of molecules, secreted factors and membrane receptors/components, made up $4 \%$ and $5 \%$ of the collection, respectively. For instance, $\operatorname{Ig} f 1$ and $\operatorname{Igf} 2$ were enriched, together with $I g f 2 r$. In addition, $F g f r l$ and Fgfr2 were all highly expressed in the undifferentiated rat ES-like cells, suggesting that these pathways could be activated in an autocrine or paracrine manner in rat ESlike cells (Figure 5C). Exogenous FGF2 is required to maintain human ES cells in the undifferentiated state [6] and IGF1 has a positive effect on human ES cell growth [25]. The expression of these ligands and receptors suggests critical roles for the endogenous IGF and FGF signaling pathways in the growth of rat ES-like cells. Further analysis of these enriched genes will probably reveal new molecular networks active in rat ES-like cells.

Signal transduction is essential for diverse biological processes. The FGF signaling pathway is known to play an essential role in maintenance of the undifferentiated state in human ES cells $[6,26]$, and self-renewal of mouse ES cells is known to be dependent upon the JAK/ STAT signaling pathway [27]. To determine the signaling pathways active in rat ES-like cells, we analyzed the KEGG pathways of the selected 629 rat transcripts 
A

\begin{tabular}{|l|l|l|l|}
\hline Gene Title & Gene Symbol & Unigene & GenBank \\
\hline procollagen, type I, alpha 2 & Col1a2 & Rn.107239 & NM_053356.1 \\
\hline inhibin beta-B & Inhbb & Rn.35074 & XM_344130.3 \\
\hline chemokine (C-C motif) ligand 2 & Ccl2 & Rn.4772 & NM_031530.1 \\
\hline procollagen, type V, alpha 2 & Col5a2 & Rn.2875 & XM_343564.3 \\
\hline twist homolog 2 (Drosophila) & Twist2 & Rn.16279 & NM_021691.2 \\
\hline Procollagen, type V, alpha 1 & Col5a1 & Rn.117 & NM_134452.1 \\
\hline chondroitin sulfate proteoglycan 2 & Cspg2 & Rn.35666 & XM_215451.4 \\
\hline glypican 3 & Gpc3 & Rn.9717 & NM_012774.1 \\
\hline cadherin 11 & Cdh11 & Rn.8900 & XM_001059464.1 \\
\hline Iroquois related homeobox 1 (Drosophila) & Irx1_predicted & Rn.46018 & XM_225101.4 \\
\hline chemokine (C-C motif) ligand 7 & Ccl7 & Rn.26815 & NM_001007612.1 \\
\hline procollagen, type 1, alpha 1 & Col1a1 & Rn.2953 & NM_053304.1 \\
\hline insulin-like growth factor 2 & Igf2 & Rn.118681 & NM_031511.1 \\
\hline transcription factor AP-2, gamma & Tcfap2c & Rn.16142 & NM_201420.2 \\
\hline EF hand domain family A1 & Efha1 & Rn.64644 & NM_134396.2 \\
\hline ceruloplasmin & Cp & Rn.32777 & NM_012532.1 \\
\hline eukaryotic translation elongation factor 2 & Eef2 & Rn.55145 & NM_017245.2 \\
\hline neurotrophin 3 & Ntf3 & Rn.9715 & NM_031073.2 \\
\hline actin, gamma, cytoplasmic 1 & Actg1 & Rn.101464 & NM_001127449.1 \\
\hline Tenascin C & Tnc & Rn.12723 & \\
\hline dihydropyrimidinase-like 3 & Dpysl3 & Rn.93365 & NM_012934.1 \\
\hline matrix metalloproteinase 16 & Mmp16 & Rn.208361 & NM_080776.1 \\
\hline cytokine receptor-like factor 1 (predicted) & Crlf1_predicted & Rn.38637 & NM_001106074.1 \\
\hline inhibin beta-A & Inhba & Rn.9874 & NM_017128.1 \\
\hline procollagen, type VIII, alpha 1 (predicted) & Col8a1_predicted & Rn.53843 & NM_001107100.1 \\
\hline paired related homeobox 2 (predicted) & Prrx2_predicted & Rn.93004 & NM_001105739.1 \\
\hline placenta-specific 1 & Plac1 & Rn.24205 & NM_001024894.1 \\
\hline glycine decarboxylase (predicted) & Gldc_predicted & Rn.171011 & NM_001107583.1 \\
\hline & & & \\
\hline
\end{tabular}

B ESTs and unknown function (48\%)

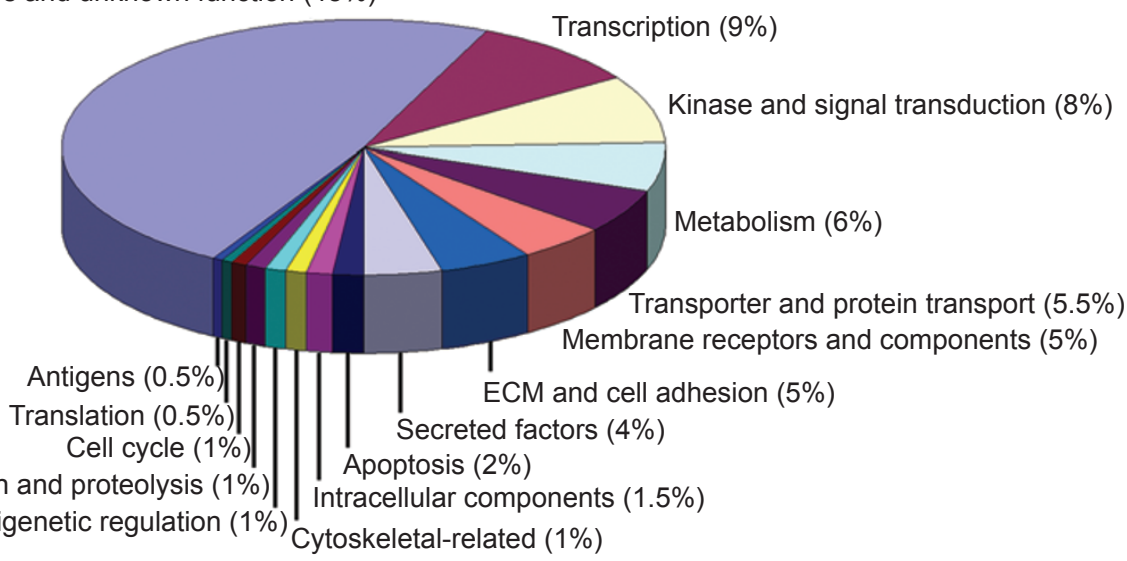

C

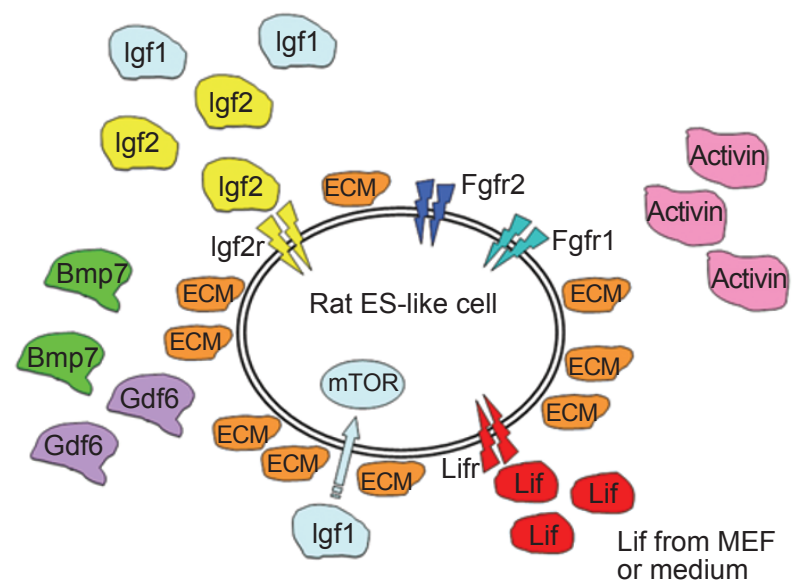

Figure 5 Functional annotation of rat ES-like cells enriched genes. (A) The enriched genes, which are expressed more than 10 -fold in the rat ES-like cells. (B) A total of 629 of the rat ES-like cells enriched transcripts are divided into 16 groups according to their functional annotation. The full list is available in the Supplementary information, Table S1. (C) Multiple signaling pathways present in rat ES-like cells. 
by DAVID tools (Supplementary information, Table $\mathrm{S} 2$ and Figure 5C). Overall, we found that the TGF $\beta /$ BMP pathway is enriched in rat ES-like cells containing components such as Dcn, Gdf6, Bmp 7, Inhbb and Inhba (Activin). This pathway has been shown to be involved in pluripotency of both mouse and human ES cells, in which Bmp is important for mouse ES cells while Activin is essential for human ES cells [20, 28, 29]. The existence of both Bmp and Activin transcripts suggests a unique signal transduction mechanism in the maintenance of rat ES-like cells. Furthermore, several transcripts of the mTOR signaling pathway (Igfl, Figf, Ddit4 and $F y b$ ) were enriched in the undifferentiated rat ES-like cells. Although the mechanism of the mTOR signaling pathway in the rat ES cells remains obscure, it is essential for proliferation of mouse ES cells and early embryos [30]. On the other hand, our analysis did not detect enrichment of the JAK/STAT signaling pathway in rat ES-like cells. However, we found that Lifr, but not Lif, was termed "present" in the rat ES-like cells. It is possible that Lif produced by the feeder layer or provided by the culture media activates Lifr present in rat ES-like cells to help maintain them in an undifferentiated state. The finding confirmed the rationality of our inclusion of LIF in the culture medium for derivation of rat ES-like cells. Another important pathway that influences the proliferation of both mouse and human ES cells is the Wnt signaling pathway $[31,32]$. Unexpectedly, the Wnt signaling pathway was not found to be enriched in the rat ES-like cells. Wnt4 was the only enriched component and it has been reported to be not essential for supporting growth of ES cells [33].

In addition to the signaling pathways, another interesting finding from our analysis was that the extracellular matrix (ECM)-receptor interaction was active in rat ESlike cells. For example, $S d c 3, F n d c 1$, Spp1, Col3a1, Col5a3, Col5a2, Col5a1, Colla1 and Colla2 were all highly expressed in these cells. The fact that human ES cells can proliferate on matrigel without feeders reflects the important role of ECM in maintaining ES cells [34]. The function of these enriched ECM transcripts is not clear, but their high level of expression supports our early observation that embryo attachment was a critical factor for derivation of the rat ES-like cells.

\section{Discussion}

Here, we report high efficiency generation of ES-like cells from rat blastocysts, which express pluripotency markers Oct4, Nanog, Tra-1-81 and AKP and produce a variety of cell types originating from all three embryonic germ layers in both EBs and teratomas. The result is sig- nificant in that this is the first report that rat ES-like cell lines are able to contribute to the three embryonic germ layers in vivo, an essential criterion in demonstrating the true pluripotent character of ES cell lines. Our result marks an important step forward in the generation of genuine rat ES cells with the capacity to produce chimeras and germ line transmissions.

We believe that one of the critical factors that allowed us to successfully produce pluripotent rat ES-like cells in this study was our identification of an optimal rat blastocyst culture medium, which promoted the attachment of cultured blastocysts to the substrate and enhanced the number of cells expressing Oct 4 in the embryos, although its exact components are not yet defined. Meanwhile, we found that the rat ES cell medium did not increase the hatching of rat blastocysts (data not shown). Many researchers have experienced considerably more differentiation of cultured rat blastocysts compared to mouse blastocysts. This could be due to the distinctive developmental potential of the early pluripotent cells between these two closely related rodents. In fact, Nichols and Buehr demonstrated that rat epiblasts produced extra-embryonic endoderm cells in culture, whereas mouse epiblasts did not [13]. Therefore, it is reasonable to expect that the longer the embryos are suspended in medium, the more differentiated cells would be produced from the epiblast. In contrast, when rat embryos attach to the substrate quickly and propagate soon after the epiblasts grow out, the undifferentiated pluripotent cells can be isolated before a massive differentiation occurs. It appears that the first $36 \mathrm{~h}$ after whole embryos are plated onto substrate is the critical period for their further development. Importantly, the early attached embryos in the rat ES cell medium express the Oct4 protein at a substantially higher level than the later attached embryos in either the mouse ES cell medium or the EScGRO. This finding is consistent with the notion proposed by Buehr et al. [8] that the continuous expression of Oct4 through the initial stages of derivation may be necessary if pluripotent cell lines are to be established.

Previously, several attempts at derivation of rat ESlike cell lines have been reported. Vassilieva et al. [12] described the establishment of rat ES-like cell lines, in which the Oct4 mRNA level was found to be lower than that of mouse ES cells. Although the cells displayed typical growth of highly compacted cell clusters, the capacity of these cells to differentiate was not presented. Later, long-term culture and differentiation of rat ES-like cells into neuronal, glial, endothelial, and hepatic lineages in vitro was reported by Ruhnke et al. [11]. However, the cells were not characterized for their expression of Oct4. Recently, Demers et al. [9] described efficient deriva- 
tion of rat ES-like cells expressing pluripotency markers. Interestingly, their ES-like cells, when injected into blastocysts, contributed to extra-embryonic tissues, but not to embryonic germ layers. Collectively, these published results have not shown that the cognate ES-like cells possess pluripotent differentiation potential in vivo. In this study, we generated six rat ES-like cell lines. Two of the lines have been extensively characterized, except for their capacity to contribute to chimeras and produce germ line transmissions, a circumstance which is now under examination in our laboratory. The establishment of such cell lines provides an opportunity for further investigation of molecular regulation in rat pluripotent cells and their potential utilization in targeted genetic modification, which could widen the application of rat disease models in biomedical research. In addition to rat ES-like cells, we also generated rat PrE cells, which can be reproducibly isolated from the differentiated rat ESlike cells. Microarray analysis showed that they express at high levels molecular markers for PrE cells, such as Gata6, Gata4, Lamb1, Sox7, Sox17 and Foxa2. Most convincingly, these cells display functional properties of PrE cells. Surprisingly, these PrE cells express Oct4, reminiscent of a previous report of transiently high expression of Oct4 in the PrE cells of mouse early embryos [35]. Currently, the function of Oct4 in rat PrE cells remains unclear. However, the established PrE cell line enables us to further investigate the unique molecular network operating in early rat embryos.

The failure in the establishment of true rat ES cell lines is primarily due to the lack of understanding of molecular regulation in rat pluripotent cells. Our transcriptional profiling and analysis of rat ES-like cells not only provide a list of the transcripts enriched in these undifferentiated cells but also suggest that multiple signaling pathways, such as those of TGF $\beta$, mTOR, IGF, FGF and ECM-receptor interaction, might be involved in maintaining self-renewal and pluripotency of the undifferentiated rat ES-like cells. In addition, LIF is perhaps a very important factor for rat ES cells. Our results indicate that rat ES-like cells share some characteristics with either mouse or human ES cells at the molecular level, while they also have their own unique transcriptional landscape. In the 629 enriched transcripts of the rat ES-like cells, few of them have been reported to be involved in maintaining pluripotency of ES cells. However, it should be noted that several core pluripotency genes such as Oct4 and Nanog were not included in the probes of the rat expression array 2302.0 Chip, although our immunostaining experiments showed their specific expression in rat ES-like cells. The apparent lack of enrichment of previously known pluripotency-related genes could have resulted from the shortage of rat genome information and lack of annotation for many transcripts. It is also highly possible that rat ES cells have their own distinctive regulatory mechanisms for maintaining self-renewal and pluripotency. Taken together, our data provide a list of uniquely expressed genes and important pathways in rat ES-like cells for further in-depth investigation. Identification of these molecular features will help us to optimize conditions for derivation and culture of true rat ES cell lines. In addition, we obtained microarray data from REF cells and compared them to those collected from rat ES-like cells. Such a comparison led to the enrichment of many endoderm marker genes for the rat ES-like cells, including Foxa2, Gata6, Gata4, Sox7, Sox17, Ihh, Hnf4 $\alpha, K r t 1-19$ and Krt1-18. Further analysis indicated that these genes are highly expressed in both our rat ES-like cells and rat PrE cells. To obtain the transcripts uniquely expressed in the undifferentiated rat ES-like cells, we thus chose the rat PrE cells as a reference, in order to preclude those genes that are also expressed in the PrE cells. However, it is worth pointing out that some known pluripotency marker genes, such as Klf4, $c-M y c$, Cdh1, Tbx3, Tbx15 and Dppa3, are expressed in both our rat ES-like cells and PrE cells. Thus, these genes are not included in our gene list of uniquely expressed genes in the undifferentiated ES-like cells when the rat PrE cells are used as a reference.

During our preparation of this manuscript, Ueda et al. [36] reported their establishment of rat ES cell lines, which could produce chimeric rats, as determined by PCR analysis of expression of a transfected GFP gene. By comparison, our study has the following advantages: i) our ES cell lines were each derived from the individual rat blastocyst, whereas the lines established by Ueda et $a l$. were generated from the pooled cells of 42 rat embryos; ii) the antibodies used in our study were verified by embryo staining and our cells were alkaline phosphatase positive, which is a characteristic of both human and mouse ES cells, while the ES cells reported by Ueda et al. were alkaline phosphatase negative; iii) importantly, our cells reproducibly generated teratomas containing various tissues from all three germ layers. In contrast, their cells produced tumors containing tissues of only two germ layers, lacking ectodermal tissue. Production of teratomas containing tissues from all three embryonic germ layers is one of the essential criteria for characterization of ES cells from any species. In addition, we derived rat ES-like cells using a new medium which enhanced rat blastocyst attachment and the generation of Oct4-positive cells. Furthermore, we report a line of PrE cells derived from our established rat ES-like cells in this study. The PrE cell line provides a better reference 
to identify genes and pathways specifically expressed in rat pluripotent cells, since PrE cells are the differentiated cell type most often seen in the culture of rat ES-like cells. In contrast, Ueda et al. used rat embryonic fibroblast cells as a reference; many endoderm marker genes were thus enriched in their rat ES cells. It should be pointed out that the PCR analysis used by Ueda et al. is not a rigorous verification of production of chimeric mice and that detection of GFP expression in various organs could have resulted from the presence of blood in the organs. Thus, more experiments are needed to confirm the generation of chimeric mice, especially given that their rat ES cells could not produce cell or tissue types from all three germ layers. Nevertheless, the two studies reveal that pluripotent embryonic cells can be generated from rat blastocysts under appropriate conditions and that the cell lines established independently share common properties, such as expression of pluripotency markers and Lifr, formation of EB in suspension culture and the ability to proliferate for an extended time in vitro. Both studies provide useful information for understanding the genetic basis of pluripotency and for optimization of rat ES cell culture conditions.

\section{Materials and Methods}

\section{Animals and embryos}

Rats of the Sprague-Dawley (SD) strain were purchased from Shanghai Laboratory Animal Center (SLAC) and maintained in SPF spaces with a 14-h light and 10-h dark regime. All animal procedures were performed according to the guidelines approved by the Shanghai JiaoTong University School of Medicine. Rat blastocysts of $4.5 \mathrm{dpc}$ were flushed from the uterus with KO-DMEM (Gibco) supplemented with $40 \mathrm{mM}$ HEPES (Gibco). Mouse and rat epiblasts were obtained from $6.0 \mathrm{dpc}$ and $7.5 \mathrm{dpc}$ embryos, respectively. MEF cells were prepared from ICR and KM strains at $13.5 \mathrm{dpc}$. For teratoma formation assays, 6 - to 8 -week-old SCID mice were employed.

\section{Statistical analysis of attachment ratio}

Attachment ratio of embryos was determined for whole and zona-pellucida free rat blastocysts. Whole rat blastocysts were plated onto irradiated mouse embryonic fibroblast (MEF) cells or matrigel with rat ES cell medium, which consisted of $50 \%$ mouse ES cell medium, $50 \%$ EScGRO (Millipore) and $2 \mu \mathrm{M}$ of GSK-3 $\beta$ inhibitor (BIO, gift from Wei Han). To remove the zona-pellucida, whole rat blastocysts were treated with Tyrode's solution (Sigma) for $10 \mathrm{~min}$ at $37^{\circ} \mathrm{C}$. Zona-pellucida free rat blastocysts were then plated onto matrigel covered 4-well plates with different medium. Attachment ratio was calculated at different time points.

\section{Derivation and culture of rat ES-like cells}

Whole rat blastocysts were plated onto irradiated mouse embryonic fibroblast (MEF) cells. When ICM clumps appeared, rat ES-like cells were passaged mechanically in the early passages. From the sixth passage, $0.05 \%$ trypsin (Invitrogen) was used to passage the cells every four to five days. Mouse ES cell medium consisted of 90\% DMEM (Invitrogen) supplemented with 10\% FBS (Hyclone), 100U/ml penicillin (Invitrogen), $100 \mu \mathrm{g} / \mathrm{ml}$ streptomycin (Invitrogen), $0.1 \mathrm{mM} \beta$-mercaptoethanol (Gibco), $2 \mathrm{mM}$ L-glutamine (Invitrogen) and 2000 units $/ \mathrm{ml}$ murine LIF (Chemicon). The rat ES-like cells were cryopreserved using $90 \%$ rat ES cell medium and $10 \%$ DMSO.

\section{EB formation assays}

EBs were generated by trypsinizing the rat ES-like cells and resulting single cells were cultured in suspension with EB culture medium, consisting of $90 \%$ DMEM, 10\% FBS, $100 \mathrm{U} / \mathrm{ml}$ penicillin (Invitrogen), $100 \mu \mathrm{g} / \mathrm{ml}$ streptomycin (Invitrogen), $2 \mathrm{mM}$ L-glutamine (Invitrogen) and $0.1 \mathrm{mM} \beta$-mercaptoethanol (Gibco). The medium was changed every other day.

\section{Derivation and culture of rat $\operatorname{PrE}$ cells from rat ES-like cells}

When rat ES-like cells were maintained on a feeder layer for more than seven days, endoderm-like cells appeared in the periphery of partially differentiated rat ES-like cell colonies, with the compacted undifferentiated rat ES-like cells in the center. We mechanically transferred the endoderm-like cells onto gelatin-coated culture dishes at a high density and cultured them in the mouse ES cell medium without LIF.

\section{Teratoma formation assays}

The rat ES-like cells were trypsinized and suspended at a density of $2 \times 10^{6} / 100 \mu \mathrm{l}$ in rat ES cell medium. A total of $4 \times 10^{6}$ cells were injected into muscle of the rear leg of 6-week-old male SCID mice.

\section{Chimeric EB formation assays}

The cells used in the assay were trypsinized and were dropped onto low attachment Petri dishes at a density of 300 cells $/ 50 \mu 1$. Two days later, all the chimeric EBs were collected from the dishes and embedded in O.C.T. The PrE cells were labeled with EGFP for immunostaining of PrE markers. They were additionally labeled with a red fluorescent dye (DiI) to trace their location in chimeric EBs. The undifferentiated mouse ES cells (E14T, gift from Austin Smith and Ian Chambers) and the rat PrE cells were mixed at a ratio of 2:1.

\section{Microarray analysis}

For each sample, $5 \mu \mathrm{g}$ of purified RNA was used for Affymetrix Chip analysis using GeneChip ${ }^{\circledR}$ Rat Expression Array 230 2.0. The computer data files to be used in data analysis (*.cel) were generated with the Affymetrix GeneChip Operating Software (GCOS) Version 1.4 (Affymetrix ${ }^{\circledR}$ ), using the statistical algorithm provided. The data for the probe level intensity were then transferred to the ArrayAssist ${ }^{\circledR}$ Software (StrataGene; La Jolla, CA) for further analysis. For each sample, background was removed and data were normalized in accordance with the MAS 5.0. The genes whose expression was up-regulated more than 2-fold and Affymetrix "P" call in rat ES-like cells were selected as significant genes, when compared with rat PrE cells. Two biological replicates of the rat ES-like cells and the PrE cells were used to produce microarray data. The significant genes were analyzed in the context of the KEGG pathway in order to identify active pathways in rat ES-like 
cells using DAVID 2008 (http://david.abcc.ncifcrf.gov/). For each term, the probability values were computed based upon a modified Fisher Exact test. EASE scores $<0.5$ were considered as significant categories.

\section{$R T-P C R$}

Total RNA was extracted from cells using TRIzol (Invitrogen) and transcribed into cDNA using oligo(dT)15 and ReverTra Ace reverse transcriptase (Toyobo). PCR reactions were carried out as previously described [37]. The RT-PCR primers are shown in the Supplementary information, Table S3.

\section{Immunofluorescence staining}

The staining was performed as described previously [38]. All primary antibodies listed in the Supplementary information, Table S4 were diluted in the blocking buffer and incubated overnight at $4{ }^{\circ} \mathrm{C}$. Fluorescently coupled secondary antibodies, including antirabbit FITC (Protein Tech Group, 1/100), anti-goat FITC (Jackson ImmunoResearch, 1/50), anti-rabbit Cy3 (Jackson ImmunoResearch, 1/300) and anti-mouse FITC (Jackson ImmunoResearch, $1 / 100)$, were incubated for $1 \mathrm{~h}$ at room temperature.

\section{Acknowledgments}

Authors express appreciation to Erbo Xu (Boston College) for his help in preparation of this manuscript and to Yuan Guan (Institute of Health Sciences, Shanghai Institutes for Biological Sciences, Chinese Academy of Sciences) for his help in subretinal injection. The study was supported by the financial support of the Grants from the Shanghai Science \&Technology Developmental Foundations (Grant number: 06dj14001), the National High Technology Research and Development Program of China (2006AA02Z197, 2006CB943901, 2007CB947904, and 2007CB948004), Shanghai JiaoTong University School of Medicine and Shanghai Institutes for Biological Sciences, CAS.

\section{Reference}

1 Evans MJ, Kaufman MH. Establishment in culture of pluripotential cells from mouse embryos. Nature 1981; 292:154-156.

2 Evans M, Hunter S. Source and nature of embryonic stem cells. C R Biol 2002; 325:1003-1007.

3 Brook FA, Gardner RL. The origin and efficient derivation of embryonic stem cells in the mouse. Proc Natl Acad Sci USA 1997; 94:5709-5712.

4 Chambers I, Smith A. Self-renewal of teratocarcinoma and embryonic stem cells. Oncogene 2004; 23:7150-7160.

5 Austin CP, Battey JF, Bradley A, et al. The knockout mouse project. Nat Genet 2004; 36:921-924.

6 Thomson JA, Itskovitz-Eldor J, Shapiro SS, et al. Embryonic stem cell lines derived from human blastocysts. Science 1998; 282:1145-1147.

7 Ouhibi N, Sullivan NF, English J, et al. Initial culture behaviour of rat blastocysts on selected feeder cell lines. Mol Reprod Dev 1995; 40:311-324.

8 Buehr M, Nichols J, Stenhouse F, et al. Rapid loss of Oct-4 and pluripotency in cultured rodent blastocysts and derivative cell lines. Biol Reprod 2003; 68:222-229.

9 Demers SP, Yoo JG, Lian L, et al. Rat embryonic stem-like
(ES-like) cells can contribute to extraembryonic tissues in vivo. Cloning Stem Cells 2007; 9:512-522.

10 Fandrich F, Lin X, Chai GX, et al. Preimplantation-stage stem cells induce long-term allogeneic graft acceptance without supplementary host conditioning. Nat Med 2002; 8:171-178.

11 Ruhnke M, Ungefroren $\mathrm{H}$, Zehle G, et al. Long-term culture and differentiation of rat embryonic stem cell-like cells into neuronal, glial, endothelial, and hepatic lineages. Stem Cells 2003; 21:428-436.

12 Vassilieva S, Guan K, Pich U, et al. Establishment of SSEA1- and Oct-4-expressing rat embryonic stem-like cell lines and effects of cytokines of the IL-6 family on clonal growth. Exp Cell Res 2000; 258:361-373.

13 Nichols J, Smith A, Buehr M. Rat and mouse epiblasts differ in their capacity to generate extraembryonic endoderm. Reprod Fertil Dev 1998; 10:517-525.

14 Beck S, Le Good JA, Guzman M, et al. Extraembryonic proteases regulate Nodal signalling during gastrulation. Nat Cell Biol 2002; 4:981-985.

15 Kaji K, Nichols J, Hendrich B. Mbd3, a component of the NuRD co-repressor complex, is required for development of pluripotent cells. Development 2007; 134:1123-1132.

16 Brons IG, Smithers LE, Trotter MW, et al. Derivation of pluripotent epiblast stem cells from mammalian embryos. Nature 2007; 448:191-195.

17 Narisawa S, Hasegawa H, Watanabe K, et al. Stage-specific expression of alkaline phosphatase during neural development in the mouse. Dev Dyn 1994; 201:227-235.

18 Umehara H, Kimura T, Ohtsuka S, et al. Efficient derivation of embryonic stem cells by inhibition of glycogen synthase kinase-3. Stem Cells 2007; 25:2705-2711.

19 Sato N, Meijer L, Skaltsounis L, et al. Maintenance of pluripotency in human and mouse embryonic stem cells through activation of Wnt signaling by a pharmacological GSK-3-specific inhibitor. Nat Med 2004; 10:55-63.

20 Qi X, Li TG, Hao J, et al. BMP4 supports self-renewal of embryonic stem cells by inhibiting mitogen-activated protein kinase pathways. Proc Natl Acad Sci USA 2004; 101:6027-6032.

21 Ying QL, Nichols J, Chambers I, et al. BMP induction of Id proteins suppresses differentiation and sustains embryonic stem cell self-renewal in collaboration with STAT3. Cell 2003; 115:281-292.

22 Hamazaki T, Oka M, Yamanaka S, et al. Aggregation of embryonic stem cells induces Nanog repression and primitive endoderm differentiation. J Cell Sci 2004; 117:5681-5686.

23 Rula ME, Cai KQ, Moore R, et al. Cell autonomous sorting and surface positioning in the formation of primitive endoderm in embryoid bodies. Genesis 2007; 45:327-338.

24 Jiang J, Chan YS, Loh YH, et al. A core Klf circuitry regulates self-renewal of embryonic stem cells. Nat Cell Biol 2008; 10:353-360.

25 Bendall SC, Stewart MH, Menendez P, et al. IGF and FGF cooperatively establish the regulatory stem cell niche of pluripotent human cells in vitro. Nature 2007; 448:1015-1021.

26 Dvorak P, Dvorakova D, Koskova S, et al. Expression and potential role of fibroblast growth factor 2 and its receptors in human embryonic stem cells. Stem Cells 2005; 23:1200-1211.

27 Matsuda T, Nakamura T, Nakao K, et al. STAT3 activation is sufficient to maintain an undifferentiated state of mouse em- 
bryonic stem cells. EMBO J 1999; 18:4261-4269.

28 Vallier L, Alexander M, Pedersen RA. Activin/Nodal and FGF pathways cooperate to maintain pluripotency of human embryonic stem cells. J Cell Sci 2005; 118:4495-4509.

$29 \mathrm{Xu}$ RH, Peck RM, Li DS, et al. Basic FGF and suppression of BMP signaling sustain undifferentiated proliferation of human ES cells. Nat Methods 2005; 2:185-190.

30 Gangloff YG, Mueller M, Dann SG, et al. Disruption of the mouse mTOR gene leads to early postimplantation lethality and prohibits embryonic stem cell development. Mol Cell Biol 2004; 24:9508-9516.

31 Dravid G, Ye Z, Hammond H, et al. Defining the role of Wnt/ beta-catenin signaling in the survival, proliferation, and selfrenewal of human embryonic stem cells. Stem Cells 2005; 23:1489-1501.

32 Cole MF, Johnstone SE, Newman JJ, et al. Tcf3 is an integral component of the core regulatory circuitry of embryonic stem cells. Genes Dev 2008; 22:746-755.

33 Li T, Wang S, Xie Y, et al. Homologous feeder cells support undifferentiated growth and pluripotency in monkey embryonic stem cells. Stem Cells 2005; 23:1192-1199.

$34 \mathrm{Xu} \mathrm{C}$, Inokuma MS, Denham J, et al. Feeder-free growth of undifferentiated human embryonic stem cells. Nat Biotechnol 2001; 19:971-974.

35 Palmieri SL, Peter W, Hess H, et al. Oct-4 transcription factor is differentially expressed in the mouse embryo during establishment of the first two extraembryonic cell lineages involved in implantation. Dev Biol 1994; 166:259-267.

36 Ueda S, Kawamata M, Teratani T, et al. Establishment of rat embryonic stem cells and making of chimera rats. PLOS ONE 2008; 3:e2800.

37 Zhang Z, Liao B, Xu M, et al. Post-translational modification of POU domain transcription factor Oct-4 by SUMO-1. FASEB $J$ 2007; 21:3042-3051.

$38 \mathrm{Xu} \mathrm{HM}$, Liao B, Zhang QJ, et al. Wwp2, an E3 ubiquitin ligase that targets transcription factor Oct-4 for ubiquitination. $J$ Biol Chem 2004; 279:23495-23503.

(Supplementary information is linked to the online version of the paper on the Cell Research website.) 